\title{
Scott F. Gilbert - Developmental Biology, 2010, Sinauer Associates, Inc., Sunderland, MA Ninth Edition
}

DOI: $10.1134 / \mathrm{S} 1062360411050043$

The ninth edition of Scott F. Gilbert's book confirms its earlier established reputation as the most complete and qualified developmental biology textbook. It not only explains and beautifully illustrates information in practically all aspects of this scienceboth descriptive and experimental-it also contains sections that other textbooks commonly do not. This concerns the detailed chapter on developmental genetics and especially — what may be the main novelty of this edition-Chapter 4, devoted to systemic biology in its medicinal, ecological, and evolutionary aspects. The inclusion of such a section in a standard textbook is worthy of particular approval, since it explains the most important general-theoretic questions that are commonly glossed over: context-dependent properties, organization levels and so-called emergence, heterogeneous causality and integration, and modularity and roughness. As well, these seemingly abstract concepts are illustrated with clear visual examples. In this connection, however, it would be desirable to briefly mention the existence of such a powerful section of modern science as self-organization theory, which gives a rigorous formulation of these and other concepts related to them (and to give corresponding references, since there are many books in the world on this topic of varying degrees of popularity).

In general, for me, Gilbert's book is primarily more an aid for teachers than for students per se: bearing in mind the comparatively small amount of time given to developmental biology even at the best universities, it is neither possible nor necessary for lectures to cover all the sections in this textbook. All the more so, they need not be explained in the order in which the textbook presents them. For instance, information contained in the chapter on cell communications should be incorporated into those sections of the course that discuss developmental stages at which these communications are particularly visible. This likewise refers to developmental genetics and, of course, to systemic biology problems: it is important that they not just float around somewhere, but that they be tied as closely as possible to specific material. I would also especially like to note the brief but precise presentation of the main historical milestones of embryological ideas.

Although the amount of factual material is quite large, certain omissions are readily noticeable. For instance, in my opinion, the section on evolutionary embryology begs for the recent work of Yu.A. Kraus et al. ${ }^{1}$, who discovered a Spemann type of embryonic induction in coral polyps. Since the chapter of systemic biology mentions P. Weiss in connection with the holistic approach, it should also mention A.G. Gurvich, whom Weiss considered his teacher and whose contribution to the theory of development was highly valued by Gilbert himself ${ }^{2}$. Similarly, for the mentioning of the embryonic character of cancer cells, it would not hurt to reference the works of G.I. Abelev et al., who were the first to describe this phenomenon.

It would be desirable for this remarkable textbook to become in one form or another available to the widest range of developmental biology teachers and students in Russia.

\section{L.V. Belousov}

\footnotetext{
${ }^{1}$ The Blastoporal Organizer of a Sea Anemone, Curr. Biol., 2007, 17(20), pp. 874-876.

2 Int. J. Dev. Biol., 1997, 41, p. 778.
} 